\title{
LAS AUTOINSTRUCCIONES Y LA PREVENCIÓN DE RESPUESTA COMO TRATAMIENTO BREVE EN UN CASO DE HIPOCONDRÍA
}

\author{
Cristina Robert Flors' \\ Psicóloga. Unidad de Salud Mental de Vila-real (Castellón)
}

\begin{abstract}
RESUMEN
La hipocondría es uno de los trastomos psiquiátricos que tiene una menor representación entre las publicaciones sobre tratamiento, a pesar de su incidencia, sobre todo entre los pacientes que acuden a las consultas de atención primaria y especializada de salud. En este trabajo se presenta el tratamiento de una mujer de 38 anos, de nivel sociocultural bajo, que acude por un trastomo hipocondríaco de 19 años de evolución. El tratamiento psicológico tuvo una duración de tres sesiones, y consistió en técnicas de autoinstrucción y prevención de respuesta. Se presentan los datos al mes, tres meses y cuatro meses del alta.
\end{abstract}

Palabras clave: Hipocondria, Tratamiento Psicológico.

\begin{abstract}
Hypochondriasis is one of the psychiatric disorders less representated in the treatment literature despite its incidence, mainly in primary and specialized health care. The case of a 38 years-old women, from a low social and cultural status, with a history of 19 years of hypochondriasis is presented. A psychological treatment based on self-instructions and response prevention was carried out in three sesions for a period of six weeks. Results are feedback presented at one, three and four months after the end of the treatment.
\end{abstract}

Koy words: Hypochondriasis, Psychological Treatment.

' Correspondencia: Cristina Robert Flors. Unidad de Salud Mental. Centro de Salud Carinena. Avda. Illes Columbretes $s / n$. Vila-real (Castellón). Espana. 


\section{INTRODUCCIÓN}

La hipocondría, tal y como la define el DSMIV (1994), consiste en una preocupación por el miedo a tener, o la convicción de tener una enfermedad grave, a partir de la interpretación personal de sintomas somáticos. Esta preocupación es persistente (más de seis meses) y se mantiene a pesar de las exploraciones y explicaciones médicas apropiadas. La persona que padece este trastorno presta atención a funciones corporales, sensaciones fisicas vagas o ambiguas o anormalidades físicas menores, se preocupa por su significado y tiende a interpretarlas como señal de la enfermedad temida. Muchas de estas personas acuden a diversos médicos y se someten a distintas pruebas diagnósticas, pero sus preocupaciones no desaparecen, a pesar de los resultados negativos y de las explicaciones recibidas. Otras personas acuden con temor a los médicos, e incluso tienden a evitarlos, por miedo a que realmente les encuentren "algo malo". La preocupación por la enfermedad se convierte en el centro de su vida, y el malestar que de ella se deriva suele provocar deterioro en el funcionamiento cotidiano del paciente.

Uno de los aspectos más confusos en el estudio de la hipocondría es el relacionado con su diagnóstico diferencial. Aunque se ha demostrado que las actitudes hipocondriacas están relacionadas con sintomas afectivos, tanto en muestras de población normal como psiquiátrica, todavia no están claros los nexos de unión entre estos sintomas (Martínez y Belloch, 1993). Diversos autores han postulado que se tratarla de una condición secundaria a la depresión y la ansiedad (Kenyon, 1976; Marks, 1981; Fisch, 1987). A esta aseveración contribuyen el hecho de que en la depresión es frecuente la aparición de diversos sintomas físicos, así como la preocupación por el significado de los mismos. Por otra parte, muchos de los sintomas que presen$\tan$ los pacientes hipocondríacos son de ansiedad, provocados por lo general por la existencia de circunstancias estresantes. De igual modo, los síntomas ansiosos y depresivos pueden ser consecuencia de la hipocondria. Otros autores, como Kellner y cols. (1987) encontraron aspectos especificos en los pacientes hipocondriacos que los diferenciaban de los depresivos y ansiosos. Además, también resulta difícil diferenciar la hipocondría de la fobia a la enfermedad, sobre todo en aquellos pacientes donde predominan el temor y las conductas de evitación y comprobación (Marks, 1981; Warwick y Marks, 1988; Salkovskis y Warwick, 1986). Esta confusión se hace patente en los actuales sistemas de clasificación diagnóstica. En concreto, el DSM-IV (1994) incluye dentro de la hipocondría tanto "el miedo a", como "la creencia de" padecer una enfermedad grave; pero, por otro lado, incluye también este miedo dentro de las fobias especificas, como fobia a la enfermedad. Esta última edición del DSM trata de distinguir ambos trastornos, de modo que aunque en ambas condiciones aparece un temor a la enfermedad, en el caso de la hipocondría este temor va acompañado por la existencia de sintomas físicos que el paciente interpreta como señal de enfermedad. De todos modos, el diagnóstico diferencial es en ocasiones dificil, pues los limites entre ambos trastornos pueden no estar claros.

\section{MÉTODO}

\subsection{Descripción del caso}

La paciente es una mujer de 38 años, de clase social baja, casada y con dos hijos varones, uno de 15 y otro de dos años. Es la mayor de cuatro hermanos. No termino los estudios primarios y lee y escribe con dificultad. Trabaja como limpiadora en una casa. Vive en un pueblo de 18.000 habitantes. Acude en febrero de 1996 a la Unidad de Salud Mental remitida por su médico de cabecera por un cuadro de hipocondría. Viene sola a la primera entrevista y, a petición nuestra, acompañada de su marido en la segunda. De aspecto triste, contesta con pocas palabras a las preguntas que se le formulan, demostrando timidez en sus manifestaciones no verbales. Su discurso es coherente.

Respecto a los antecedentes personales, la paciente relata que el embarazo, parto y desarrolio evolutivo fueron normales. Verbaliza una buena relación familiar y adaptación escolar. Sitúa la aparición de la menarquía a los 14 años. Ha tenido dos partos normales y un aborto. En cuanto a su historial médico, relata haber sufrido cálculos de vesícula y tiene artrosis. No presenta problemas psicológicos anteriores. Desde que se casó, hace 16 años, hasta hace dos años, vivieron en la casa de sus padres. EI marido atribuye a esto gran parte de sus pro- 
blemas, pues cree que "la madre la ha tenido dominada por ser la única chica". Desde que viven de modo independiente, la paciente se muestra algo más asertiva con su madre, lo que ha mejorado la relación de pareja. Dice tener buenas relaciones laborales y sociales. Como antecedentes familiares, comenta que su madre es "nerviosa", sin poder especificar más el diagnóstico, y está en tratamiento con tranquilizantes. Un tlo materno estuvo en tratamiento psiquiátrico "por depresión".

\subsection{Motivo de consulta}

Dice "estar muy obsesionada con las enfermedades", sobre todo las relacionadas con el cáncer. El problema se inicia tras la muerte de su padre por cáncer intestinal, hace 19 años, tras un periodo largo de sufrimiento (14 meses). Ella estuvo mucho tiempo cuidándolo. Tenia muy buena relación con él (la paciente verbaliza que "era su hija favorita"). Tras su muerte, recibió tratamiento psiquiátrico durante dos o tres años por sintomas similares a los actuales. Recuerda que en aquel tiempo iba mucho al médico, por miedo a tener ella también cáncer. Ha recibido distintos tratamientos farmacológicos a lo largo de estos años, con antidepresivos y ansioliticos. Aunque han existido temporadas con mejoria en la sintomatologia, relata que "las preocupaciones no se le han ido nunca del todo". Al principio temía tener cáncer de intestino, pero en este momento le preocupa el cáncer de mama. Esta preocupación hace que "se sienta muy mal, con taquicardias, ahogos, ansiedad, pérdida de ilusión por las cosas". Dice sentirse "amargada" y perder gran parte del apetito. Desde hace unos meses estas preocupaciones se han exacerbado, a raiz de ver una pelicula (sólo el inicio) en la televisión sobre el cáncer.

Se le pauta tratamiento farmacológico con dos antidepresivos: paroxetina (Seroxat) y clorhidrato de clomipramina (Anafranil). Con el tratamiento se observa una mejoría en la sintomatologia ansioso-depresiva, sin desaparecer completamente las preocupaciones sobre el cáncer. En agosto, a los seis meses de iniciar este tratamiento, se observa una recaida, por lo que se decide completar el tratamiento farmacológico con un abordaje psicológico.

\subsection{Evaluación}

Se llevó a cabo a lo largo de dos sesiones. Como se comentó anteriormente, la primera a solas con la paciente y la segunda contando también con el marido de ésta. Teniendo en cuenta que la paciente tenia graves dificultades para leer y escribir, se redujeron al mínimo el número de cuestionarios, y éstos fueron contestados en consulta, con la ayuda del terapeuta. Se utilizaron los siguientes instrumentos:

- Entrovista clínica: proporcionó la información necesaria para desarrollar la historia clínica y evaluar las conductas objeto del tratamiento.

- Illness Behavior Questionaire (I.B.Q., Pilowsky y Spence, 1983). Evalúa siete factores y un indice general de hipocondria. Estos factores son los siguientes:

1) Hipocondria general (HG): factor general de conducta de enfermedad. Incluye preocupación y actitudes fóbicas ante la enfermedad.

2) Convicción de enfermedad (CE): preocupación por los síntomas y creencia firme en una enfermedad fisica.

3) Percepción psicológica versus somática de enfermedad (PP): tendencia a conceptualizar la enfermedad en términos psicológicos, frente a la tendencia a somatizar.

4) Inhibición afectiva (IA): dificultad para expresar a los demás los sentimientos personales, principalmente los de enfado.

5) Perturbación afectiva (PA): reconocimiento de problemas afectivos, especialmente de ansiedad y depresión.

6) Negación (NE): Tendencia a negar problemas cotidianos y/o a explicar éstos en base a sus problemas fisicos.

7) Imitabilidad (IR): Sentimientos de enfado e irritabilidad en las relaciones interpersonales.

- Valoración subjetiva del miedo y la convicción de tener cáncer: La paciente valora su grado de creencia (0-100\%) en la idea "Tengo cáncer o lo estoy desarrollando" y su miedo (en una escala de $0=$ nada a $10=$ muchísimo) a tener cáncer $\theta$ ir al médico.

- Registro de conductas de comprobación de los pechos, durante un periodo de 10 dias, de linea-base, antes de iniciar el tratamiento, y a lo largo del mismo (anexo $n^{\circ} 1$ ).

- Inventario de Autoevaluación de la Ansiodad Estado-Rasgo (STAI) (Spielberger, Gorush y Lushene, 1970), para valorar el nivel de ansiedad situacional (ansiedad-estado) y general (ansiedad-rasgo). 
Tabla 1.- Cumplimentación de instrumentos en las diversas sesiones

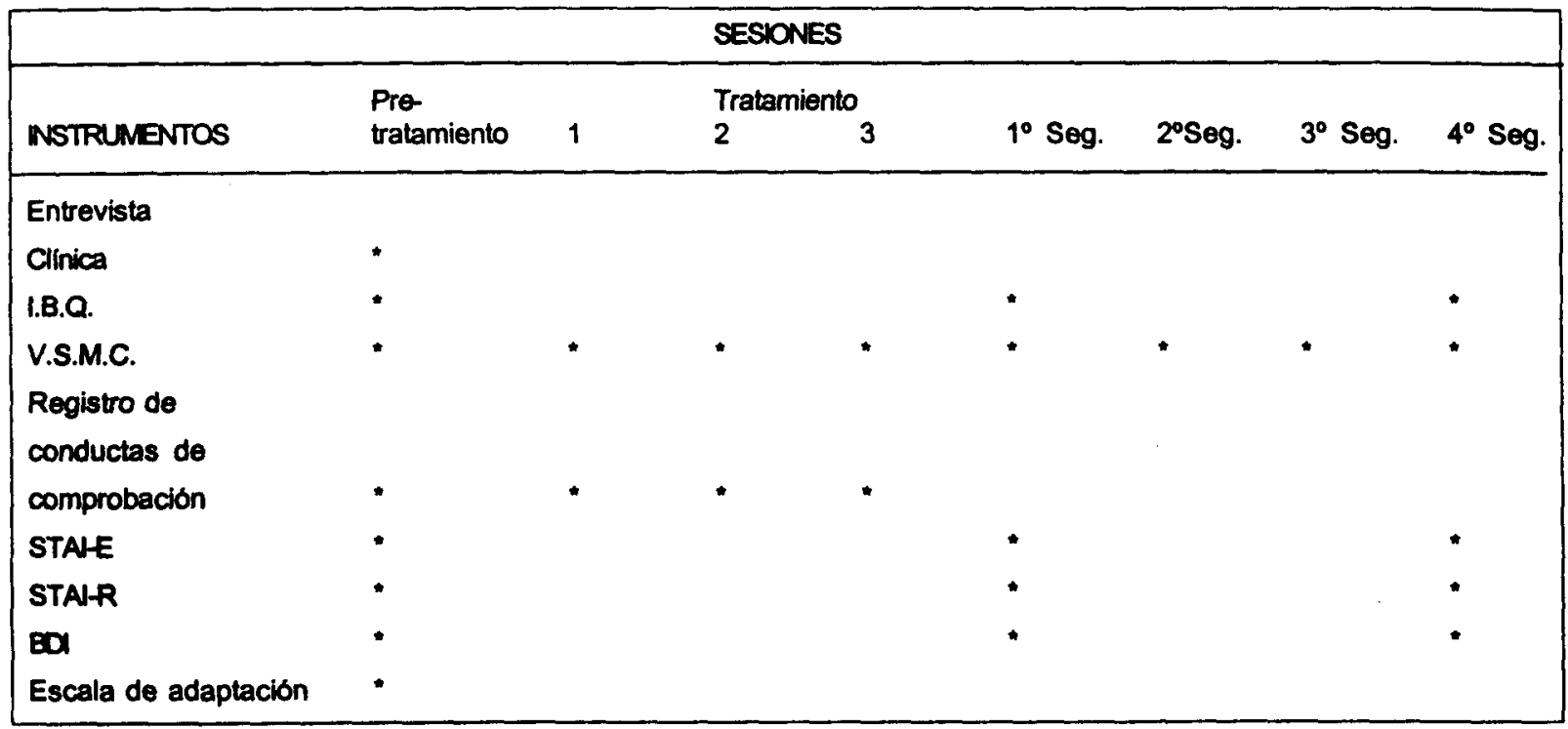

- Inventario de Depresión de Beck (B.D.I., 1961), para valorar la severidad de los sintomas depresivos.

- Escala de Adaptación, de Echeburúa y Corral (1987).

Debido a las dificultades prácticas anteriormente comentadas, no fue posible aplicar todos los instrumentos en los momentos de evaluación previamente establecidos. En la tabla $n^{\circ 1}$ puede observarse los periodos en que fueron cumplimentados cada uno de ellos.

\section{Análisis funcional}

Entre los factores predisponentes hacia la hipocondria parece encontrarse la actitud sobreprotectora por parte de uno o ambos progenitores (Starcervic, 1989), en este caso la madre de la paciente. Además, la vivencia del sufrimiento y muerte del padre de la paciente con cáncer, actuó seguramente como desencadenante del cuadro, ya que posibilitó el surgimiento del miedo (por procesos de condicionamiento clásico, aprendizaje observacional y de mediación verbal) y de sintomas depresivos, que, como ya se ha comentado, guardan gran relación con el origen y mantenimiento de los trastornos hipocondriacos. En el momento actual, el cuadro hipocondríaco viene definido por los siguientes elementos:

\section{- Situaciones desencadenantes}

1) Ver un programa en TV o escuchar noticias sobre enfermedades (principalmente cáncer).

2) Tener que ir al médico.

3) Observar algún cambio en su cuerpo, sobre todo algún pequeño bulto en el pecho.

\section{- Respuestas cognitivas:}

1) Auto-atención hacia el propio cuerpo: reconoce en ella los sintomas que ha escuchado.

2) Piensa que ella puede tenerto y que los médicos no se den cuenta.

3) Imágenes sobre lo que tener cáncer supondría (la operación, el quitarle el pecho ).

4) Pensamientos como "La pérdida de peso puede ser un sintoma del cáncer".

5) Miedo a que los médicos puedan descubrirle alguna enfermedad.

6) Pesadillas donde aparece ella hospitalizada, o donde ve a su padre y ella sabe que va a morir, y al final, éste se muere. Estas pesadillas ocurren casi a diario.

\section{- Respuestas emocionales y fisiológicas:}

Ansiedad (taquicardia y sensación de ahogo), tristeza y perdida de peso. 


\section{- Respuestas motoras:}

1) De escape: apaga la TV o cambia de tema.

2) De evitación: evita ir al médico.

3) De comprobación: con frecuencia se palpa los pechos, para ver si encuentra un bulto. Cuando encuentra alguno, lo aprieta con fuerza.

Pregunta con frecuencia a una compañera de trabajo, que le tranquiliza, diciéndole que no tiene nada.

\section{- Consecuencias:}

1) Alivio de la ansiedad cuando escapa o evita las situaciones que le producen ansiedad (refuerzo negativo).

2) Alivio de la ansiedad cuando su companera o marido le tranquilizan.

3) Señales o glándulas inflamadas en el pecho, consecuencia de apretarlas con fuerza en sus conductas de comprobación.

4) Aumento de la ansiedad a consecuencia de las conductas de comprobación cuando encuentra un bulto. Disminución cuando no encuentra señales sospechosas.

\subsection{Deterioros asociados al problema actual}

Para valorar este punto, se le pasó la Escala de Adaptación de Echeburúa y Corral (1987), obteniéndose los siguientes resultados:

- Area laboral: inexistente.

- Area social: leve. Cuando se siente más preocupada, disminuye el deseo de relacionarse socialmente y el placer que estos encuentros le proporcionan.

- Area de tiempo libre: leve.

- Area de pareja: grave. No siente apoyo por parte de su marido: Éste dice "no creer en psicólogos" y que "todo son tonterías". Abuso de alcohol crónico por parte del marido, lo que provoca frecuentes discusiones entre ambos. En el momento actual este consumo ha disminuido en gran medida, por lo que se observa una mejora en la relación de pareja.

- Area familiar: moderado.

- Area personal: teniendo en cuenta todos los aspectos anteriores, la paciente señala como graves las repercusiones que este trastorno tiene para su vida.

\subsection{Otros problemas}

Parece presentar una baja autoestima: el marido comenta que "se ve inferior a los demás", sobre todo en el aspecto intelectual, lo que confirma la paciente. Esta baja autoestima se acompaña de un déficit en asertividad: ambos informan que a la paciente le cuesta "contestar" a las personas, sobre todo si no son de confianza. Además, tiende a evitar las situaciones de confrontación (ej.: si tiene que devolver algo, manda al marido para que éste lo haga). Se ve a si misma como una persona tímida, sensible, rencorosa (le cuesta meses olvidar las cosas) y algo envidiosa (de los bienes materiales de los demás). La paciente presenta rasgos de ansiedad generalizada, como un miedo frecuente sobre la posibilidad de que su familia, principalmente sus hijos, puedan tener un accidente o enfermedad grave. También comenta la existencia de dificultades en la relación con su hijo de dos años, ya que éste parece presentar síntomas de hiperactividad. Además, relata la existencia de problemas económicos. El marido lleva un mes en el paro. De momento cobra el subsidio de desempleo y está pendiente de que le llamen para un posible trabajo.

\subsection{Psicopatograma}

No presenta alteraciones del lenguaje, estado de conciencia, curso del pensamiento, memoria, percepción, orientación, inteligencia, ni juicio y creencias. Respecto al contenido del pensamiento, se evidencia la existencia de preocupaciones constantes sobre la posibilidad de padecer cáncer. Existe conciencia de enfermedad.

\subsection{Diagnóstico}

Según el sistema de clasificación diagnóstica del DSM-IV (American Psychiatric Association, 1994):

Eje 1: F45.2 Hipocondria [300.7]

Eje 2: Z 03.2 No hay diagnóstico. [V.7109]

Eje 3: Ninguno.

Eje 4: Conflictos en la relación de pareja. Dificultades en la relación con un hijo.

Problemas económicos.

Eje 5: $\quad E E A G=60$ 


\section{TRATAMIENTO}

La terapia psicológica se inició a finales de octubre de 1996. Tuvo una duración de tres sesiones. La primera de una hora de duración, y las dos restantes de 30-45 minutos. La primera y segunda sesión estuvieron separadas por un periodo de dos semanas. Debido a la práctica desaparición de los sintomas tras esta segunda sesión, la tercera se llevó a cabo un mes después, donde se dió por finalizado el tratamiento. El primer seguimiento tuvo lugar al mes del alta; el segundo seguimiento se llevó a cabo a los tres meses de finalizado el tratamiento; el tercero a los cuatro meses; y el cuarto y último seguimiento a los siete meses de finalizado el tratamiento. A lo largo de toda la terapia, asi como en los seguimientos, se mantuvo el tratamiento farmacológico, iniciado en febrero de 1996. Al inicio de la terapia, las dosis eran de $20 \mathrm{mg}$. de paroxetina y $37.5 \mathrm{mg}$. de clorhidrato de clomipramina. Se mantiene asi hasta el primer seguimiento del tratamiento psicológico, en que se reduce la clomipramina a $25 \mathrm{mg}$. por día. En el tercer seguimiento, se reduce la paroxetina a $10 \mathrm{mg}$. diarios. En el último seguimiento se le pauta una disminución gradual de la paroxetina (5 mg. diarios durante 15 dias $y$, posteriormente, eliminarlo).

El tratamiento psicológico se adaptó a partir del propuesto por Salkovskis y Warwick (Salkovskis, 1989; Warwick, 1989; Warwick y Salkovskis, $1989 ; 1990$ ) y estuvo compuesto de los siguientes componentes, que se pactaron con la paciente y el marido y se les enseñaron en la primera sesion:

Componente educativo: Se le explicó qué es la ansiedad, cómo se manifiesta en los tres sistemas de respuesta, el papel desencadenante de los pensamientos atemorizadores y cómo podia debatirlos, y el papel que juegan las conductas de comprobación en el mantenimiento del trastorno. Como ayuda, se le leyó un extracto del apartado "Autoexploración de los pechos" del manual "La consulta ginecologica", editado por el Ministerio de Asuntos Sociales y el Ministerio de Sanidad y Consumo (anexo $n^{\circ} 2$ ), haciendo especial hincapié en la normalidad de encontrar pequeñas protuberancias en el pecho antes de la menstruación, y la necesidad de autoexplorar los pechos sólo después de la misma.
Prevención de respuesta: Se le pidió que se autoexplorara los pechos sólo 2-3 dias después de la menstruación y una sola vez, indicándole cómo hacerlo (de forma suave, sin apretar de modo brusco). Acudir al médico sólo si se mantenian los bultos por más de dos ciclos consecutivos. Debla también eliminar las preguntas de comprobación sobre el cáncer, especialmente las que dirigia a su compañera de trabajo. Al marido se le ensenó a cómo responder a sus preocupaciones (no tranquilizarla y recordarle que pusiera en marcha lo que se le había enseñado en la terapia).

Componente cognitivo: Cada vez que le asaltara una preocupación sobre el cáncer, debía darse una respuesta racional ("No tengo motivos para preocuparme. Este pensamiento sólo me sirve para sentirme mal"). En caso de que notara bultos en la autoexploración, recordaría que es algo normal, según se le habla explicado. Si éstos se mantenlan por más de dos ciclos, debia recordar que en el $80 \%$ de los casos son problemas benignos. Se le hizo repetir esta información hasta que se comprobó que la paciente la habla comprendido y memorizado.

Técnicas de distracción: Se pactaron con ella algunas actividades distractoras (hacer una faena de casa, cantar una canción, hablar de algún tema no relacionado con la enfermedad) que llevaría a cabo cada vez que se iniciara una preocupación sobre el cáncer, tras haberse dado una respuesta racional.

Las siguientes sesiones se dedicaron a revisar las tareas, comentar las dificultades y reforzar el progreso.

\section{RESULTADOS}

Como se observa en la tabla $n^{\circ} 2$, el síntoma principal de la paciente consistla en un miedo a desarrollar cáncer, valorado con un 10 de miedo en una escala de $0-10$, asi como un miedo a acudir a los médicos (valorado con un 8 usando la misma escala). Ambos miedos disminuyeron en gran medida a la terminación del tratamiento, y la mejoría se mantenía en los distintos seguimientos. En segundo lugar, aunque la paciente no estaba siempre convencida de tener un cáncer, esta creencia era mayor cuando detectaba algún signo de posible enfermedad (un pequeño bulto en el pecho), valorando su grado de convencimiento en padecer dicha 
Tabla 2.- Valoración subjetiva del miedo y la convicción de tener cáncer

\begin{tabular}{|c|c|c|c|c|c|}
\hline & PRE-TERAPIA & 19SEG. & $2^{\circ}$ SEG. & $3^{\circ}$ SEG. & $4^{\circ}$ SEG. \\
\hline $\begin{array}{l}\text { Grado de creencla } \\
\text { en la idea } \\
\text { "Tengo cáncer o lo estoy } \\
\text { desarrollando". (de } 0-100 \% \text { ) }\end{array}$ & 60 & 10 & 5 & 5 & 0 \\
\hline $\begin{array}{l}\text { Mledo a tener cáncer. } \\
\text { (de 0-10) }\end{array}$ & 10 & 2 & 2 & 1 & 1 \\
\hline $\begin{array}{l}\text { Miedo a ir al médico. } \\
\text { (de 0-10) }\end{array}$ & 8 & 2 & 0 & 1 & 1 \\
\hline
\end{tabular}

Tabla 3.- Puntuaciones obtenidas en el Cuestionario de Conducta de Enfermedad (Pilowsky, 1977).

\begin{tabular}{|lccc|}
\hline & PRE-TERAPIA & 19SEGUIMIENTO & 40 SEGUIMIENTO \\
\hline H.G. & 5 & 5 & 2 \\
C.E. & 5 & 5 & 0 \\
P.P. & 2 & 3 & 3 \\
I.A & 0 & 2 & 1 \\
P.A. & 5 & 2 & 2 \\
N.E. & 1 & 3 & 2 \\
I.R & 2 & 0 & 0 \\
L.H. & 12 & 9 & \\
\hline H.G.= Hipocondria General & & P.A.= Perturbación Afectiva. \\
C.E.= Convicción de Enfermedad & & N.E.= Negación. \\
P.P.= Percepción Psicológica vs & & I.R.= Irritabilidad. \\
Somática de la Enfermedad & I.H.= Indice Whiteley de Hipocondria. \\
I.A.= Inhibición Afectiva &
\end{tabular}

enfermedad antes del tratamiento en un $60 \%$. Esta creencia se ve modificada al finalizar el tratamiento y mucho más en los seguimientos ( $5 \%$ en los dos seguimientos siguientes y $0 \%$ en la última visita).

Si analizamos los resultados obtenidos en el Cuestionario de Conducta de Enfermedad (Pilowsky y Spence, 1983) observamos que los factores más directamente relacionados con la hipocondria, apenas se vieron modificados al finalizar la terapia. Como puede observarse en la tabla $n^{\circ} 3$, las puntuaciones en los factores "Convicción de enfermedad" e "Hipocondria General" fueron las más elevadas antes de iniciar el tratamiento y las que menos se ven afectadas por el mismo. Sólo se observa una ligera reducción en la "Perturbación Afectiva", en el factor de "Irritabilidad" y en el "Indice Whiteley de Hipocondria". Se puede apreciar un ligero aumento en la puntuación de los factores "Percepción Psicológica vs Somática de la Enfermedad" y en el de "Negación". La puntuación obtenida poco después de finalizar el tratamiento en los dos primeros factores, junto con la del I.H., que todavia es elevada, podria hacernos suponer que su preocupación por los síntomas y su convicción de padecer una enfermedad apenas se ha visto modificada con el tratamiento, haciendo probable una recaida futura en sus temores y conductas hipocondriacas. 
Sin embargo, siete meses después de finalizar el tratamiento, en el último seguimiento, disminuyeron las puntuaciones de la paciente en todos los factores, incluso en los que habian permanecido más estables en el primer seguimiento. De este modo, la puntuación en los factores más directamente relacionados con la hipocondria, I.H. y C.E. es de cero o cercana a cero, y se aprecia una importante disminución en la puntuación del factor H.G..

Por otro lado, y como se aprecia en la tabla $n^{\circ} 4$, se observa un cumplimiento exacto de la prevención de respuesta de comprobación, que se mantiene en todos los seguimientos, pues la paciente pasa de varias (2-3) comprobaciones de los pechos al dia, realizadas de manera incorrecta (apretando hasta que se producia dolor e irritaciones), a una comprobación al mes, realizada después de la menstruación y de manera correcta. Asimismo, la paciente manifestó desde el primer momento haber disminuido en gran medida la búsqueda de tranquilización en otras personas $y$, cuando no le fue posible, tanto su compañera de trabajo como su marido (esto último fue confirmado por él mismo), evitaron tranquilizarle.
En las variables relacionadas con la ansiedad y el estado de ánimo (tabla $n^{\circ} 5$ ), la paciente obtuvo en el B.D.I. una puntuación de 15, lo que indica un nivel de depresión media (disforia). En los seguimientos estos sintomas han disminuido, hasta el grado de no depresión. Esta mejoria en el estado de ánimo podria explicar la disminución observada en la puntuación en los factores de "Perturbación Afectiva" e "Irritabilidad" del I.B.Q. En cuanto a la ansiedad, evaluada por el STAI, el grado de ansiedad-estado antes del tratamiento era elevado (centil 80 ), para verse reducido a la media (centil 40-45) en los distintos seguimientos, a pesar de los problemas económicos existentes. Respecto a la ansiedad-rasgo, también presentaba una ansiedad elevada antes de iniciar el tratamiento (centil 70). La reducción observada en ésta tras el tratamiento (centil 61) es inferior a la experimentada en la ansiedad-estado. Sin embargo, esta disminución se acentúa en el último seguimiento (centil 4), lo que podria apoyar la existencia de cambios más estables en el estado de ánimo.

Tanto en el momento del alta como en los diversos seguimientos, la paciente informa de una desaparición casi total de las preocupacio-

Tabla 4.- Número de comprobaciones (palpaciones de pecho) diarias

\begin{tabular}{|lllll|}
\hline PRE-TERAPIA & 1०SEG. & $2^{\circ}$ SEG. & $3^{\circ} \mathrm{SEG}$. & $4^{\circ} \mathrm{SEG}$. \\
$2-3$ al día & 1 al mes & 1 al mes & 1 al mes & 1 al mes \\
\hline
\end{tabular}

Tabla 5.- Puntuaciones en los distintos cuestionarios antes de iniciar la terapia y en los distintos seguimientos

\section{PRE-TERAPIA}

15

35

28
1॰ SEG.

4

17

25 $4^{\circ}$ SEG.

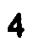

18

10 
nes sobre el cáncer. Si alguna vez han aparecido, dice haberlas controlado rápidamente dándose una respuesta racional, seguida por la búsqueda de alguna distracción. La paciente relata como situación más difícil la relacionada con la aparición de dolor en los pechos dias antes de la última regla, pero se muestra satisfecha porque fue capaz de trabajarla y de controlar la ansiedad. En todas las sesiones tras el alta, la paciente informa de una ligera ansiedad, relacionada con la situación de paro en la que se ha quedado su marido, lo que les sitúa en una posición económica muy precaria. Hasta el momento actual, trata de no "dramatizar" e intentar buscar soluciones para hacer frente a los gastos más inminentes.

\section{CONCLUSIONES}

Hemos podido ver a lo largo de la descripción del caso que los sintomas hipocondrlacos se desencadenan al inicio de la vida adulta, a partir de la enfermedad grave y muerte de una persona muy cercana a la paciente (su padre). Estos sintomas presentaban un curso crónico, con periodos de mayor y menor intensidad a lo largo de 19 años, siendo acompañados por síntomas de ansiedad $y$, en menor medida, depresivos. Esto se corresponde con la descripción hecha por la A.P.A. y otros autores (APA, 1994; Avia, 1993; Warwick y Salkovskis, 1990). Los resultados, presentados en el apartado anterior, muestran como la terapia, de breve duración, consigue modificar tanto los sintomas hipocondriacos como los emocionales. Algunos de estos cambios son visibles al poco de finalizar el tratamiento, mientras que otros sólo se hacen patentes tiempo después. Estos resultados coinciden con los obtenidos en otros estudios (Martinez y Botella, 1995) e indican la necesidad de prolongar los seguimientos, a fin de observar la evolución que se produce con el paso del tiempo. Esto es especialmente importante en los tratamientos breves, ya que en tan corto período de tiempo, aunque el tratamiento haya sido eficaz, el cambio puede no ser evidente en aquellos factores más estables. Así, podriamos sacar la falsa conclusión de que éstos no se han visto alterados por la terapia, cuando los cambios podrian evidenciarse a más largo plazo.
Otro aspecto importante a resaltar obedece a la distinción entre hipocondría y fobia específica a las enfermedades. Como comentamos en la introducción, esta distinción resulta confusa en el D.S.M.-IV (A.P.A., 1994). Como señalan Martinez y Belloch (1993), parece tratarse de dos entidades diferentes: en el segundo caso predomina el miedo a desarrollar una enfermedad, mientras que en la hipocondria la persona se preocupa por padecerla ya. Pero a veces, como en este caso, puede resultar dificil realizar el diagnóstico diferencial, pues el paciente alterna el miedo a llegar a desarrollar una enfermedad (el cáncer), con la convicción de tenerla ya. En nuestra paciente, este grado de convicción era mayor cuantos más síntomas físicos (pequeños bultos o irregularidades en las mamas) detectaba. También hemos visto cómo, en estas ocasiones, la paciente entraba en un círculo vicioso, donde las comprobaciones (autoexploraciones agresivas de los pechos) daban lugar a signos físicos que eran malinterpretados por la paciente como señal de enfermedad, lo que, a su vez, daba lugar a más comprobaciones. De todos modos, creemos que esta paciente se enmarcaria mejor dentro del subgrupo de pacientes hipocondriacos donde predomina el componente fóbico-obsesivo (Avia, 1993; Marks, 1987), lo cual podria explicar el buen resultado obtenido con las técnicas de terapia más eficaces en estos trastomos. En este tipo de personas, el tratamiento debería constar de, al menos, dos componentes: uno, cognitivo, centrado en la modificación de las interpretaciones de las sensaciones corporales (House, 1989; Salkovskis, 1989; Wanwick, 1989; Warwick y Salkovskis, 1989; 1990); otro, consistente en técnicas de exposición y prevención de respuesta, incluyendo la eliminación de búsqueda de información tranquilizadora (Logsdail, Lovell, Warwick y Marks, 1991; Martinez y Botella, 1995; Salkovskis y Warwick, 1986; Visser y Bouman, 1992; Villalobos, 1992; Warwick y Marks, 1988).

Analizando los resultados obtenidos en la terapia, llama la atención la mejoría experimentada por la paciente tras un número tan limitado de sesiones, a pesar de la cronicidad del cuadro. Una de las posibles explicaciones hace referencia a la definición dada por Pilowsky (1987) sobre "conducta de enfermedad". Según este autor, no deberiamos diagnosticar hipocondria 
mientras el paciente no haya recibido una explicación satisfactoria, adecuada a su edad y nivel socioeducativo y cultural, de sus síntomas. Pensamos que en este caso uno de los elementos decisivos en la terapia fue la explicación razonada, y apoyada con textos, sobre la normalidad de la existencia de pequenos bultos en el pecho en las mujeres antes de la menstruación, ya que la paciente refirió no conocer este aspecto y quedó muy asombrada de que esto fuera "habitual".

Para finalizar, recalcar la enorme importancia de adecuar las técnicas de terapia a cada caso individual, teniendo en cuenta el análisis funcional y las caracteristicas socioculturales de cada paciente. Además, y en casos como el presente, de larga evolución, es necesario realizar seguimientos a largo plazo a fin de valorar la eficacia real del tratamiento y prever posibles recaidas. Es interesante resaltar cómo la práctica constante de las habilidades aprendidas produce, a los siete meses de finalizado el tratamiento, una notable disminución en los sintomas hipocondriacos y en la convicción de enfermedad, asi como en la percepción que la paciente tiene sobre su estado de ánimo.

\section{REFERENCIAS BIBLIOGRAFICAS}

American Psychiatric Association (1994): Diagnostic and Statistical Manual of Mental Disorders: DSM-IV. (en castellano, MASSON, S.A., Barcelona, 1995).

Avia, M.D. (1993): Hipocondria. Barcelona, Martinez Roca.

Beck, A. (1961): An inventory for measurement of depression. Archives of General Psychiatry, A, 561-571.

Echeburúa, E. y Corral, P. (1987): Escala de Adaptación. Manuscrito no publicado.

Fisch, R. (1987): Masked depression: its interrelations with somatization, hypochondriasis and conversion. Intemational Joumal of Psychiatry in Modicine, 17, 367379.

House, A. (1989): Hypochondriasis and related disorders: assessment and management of patients referred for a psychiatric opinion. General Hospital Psychiatry, 11, 156-165.
Kellner, R.; Abbott, P.; Winston, M. y Pathak, D. (1987): Fears, beliefs and attitudes in DSM-III hypochondriasis. The Journal of Nervous and Mental Disease, 175, 20-25.

Kenyon, F. (1976): Hypochondriacal states. British Journal of Psychiatry, 129, 1-14.

Logsdail, S.; Lovell, K.; Warwick, H.M.C. y Marks, I. (1991): Behavioural treatment of AIDSfocused illness phobia. British Journal of Psychiatry, 159, 422-425.

Marks, I. (1981): Cure and care of neurosis. New York, Wiley. (en castellano, Barcelona, Martínez Roca, 1991).

Marks, I. (1987): Fears, phobias, and rituals. Oxford, Oxford University Press. (en castellano, Barcelona, Martínez Roca, 1991).

Martinez, M.P. y Belloch, A. (1993): Relaciones de los miedos y creencias hipocondriacas con ansiedad, depresión y sintomas somáticos. Análisis y Modificación de Conducta, 19, 461-478.

Martínez, M.P. y Botella, C. (1995): Aplicación de un tratamiento cognitivo-conductual a un caso de hipocondria primaria. Análisis y Modificación de Conducta, 21, 697-734.

Pilowsky, I. (1987): Abnormal iliness behaviours: a review of the concepts and its implications. En S. McHugh y M. Vallis (Eds.): Illness Behavior. New York, Pergamon.

Pilowsky, I. y Spence, N. (1983): Manual for the IIIness Behaviour Questionnaire (IBQ). Adelaida, Australia. Royal Adelaide Hospital. Salkovskis, P.M. (1989): Somatic problems. En K. Hawton, P.M. Salkovskis, J. Kirk y D.M. Clark (Eds.): Cognitive-behaviour therapy for psychiatric problems: a practical guide. Oxford: Oxford University.

Salkovskis, P.M. y Warwick, H.M.C. (1986): Morbid preoccupations health anxiety and reassurance: a cognitive-behavioural approach th hypochondriasis. Behaviour Research and Therapy, 24, 597-602.

Spielberger, C.D.; Gorush, R.L. y Lushene, R.D. (1970): Manual for the state-trait inventory. Palo Alto, California. Consulting Psychologist Press.

Starcevic, V. (1989): Pathological fear of death, panic attacks and hypochondriasis. The American Joumal of Psychoanalysis, 49, 347-362.

Villalobos, A. (1992): Tratamiento conductual de un caso de hipocondría. Analisis y Modificación de Conducta, 18, 53-61. 
Visser, S. y Bouman, T.K. (1992): Cognitivebehavioural approaches in the treatment of hypochondriasis: sex single case cross-over studies. Behaviour Research and Therapy, 30, 301-306.

Warwick, H.M.C. (1989): A cognitive-behavioural approach to hipochondriasis and health anxiety. Joumal of Psychosomatic Research, 33, 705-711.

Warwick, H.M.C. y Marks, I. (1988): Behavioural treatment of illness phobia and hypochon- driasis. A pilot study of 17 cases. British Journal of Psychiatry, 152, 239-241.

Warwick, H.M.C. y Salkovskis, P. (1989): Hypochondriasis. En J. Scott, J.M.G. Williams y A.T. Beck (Eds.): Cognitive therapy in clinical practice: an illustrative casebook. Londres: Routledge.

Warwick, H.M.C. y Salkovskis, P. (1990): Hipochondriasis. Behaviour Research and Therapy, 28, 105-117.

ANEXO 1

REGISTRO DE CONDUCTAS DE COMPROBACIÓN

\begin{tabular}{|l|l|l|}
\hline DIA & $\begin{array}{l}\text { NÚMERO DE VECES QUE TOCO LOS PECHOS, } \\
\text { PARA COMPROBAR COMO ESTAN. } \\
\text { (HACER UNA X POR CADA VEZ) }\end{array}$ & $\begin{array}{l}\text { NÚMERO TOTAL } \\
\text { DE VECES } \\
\text { (SUMO LAS X) }\end{array}$ \\
\hline & & \\
\hline
\end{tabular}

\section{ANEXO 2}

\section{INFORMACION PROPORCIONADA A LA PACIENTE SOBRE LA AUTOEXPLORACION DE LOS PECHOS (1)}

Muchas mujeres antes de cada regla notan hinchazón o dolor en los pechos. También en otras es frecuente tener una cierta cantidad de protuberancias. Si no estamos acostumbradas a palpamos los pechos después de cada regla esto puede producimos desconcierto o miedo.

Si aún no has aprendido a hacerlo, puedes comenzar unos dias después de la regla, porque tus pechos estarán más pequeños. Acostúmbrate a conocer y palpar su tamaño y sus cambios de forma.

(1) Extraido del folleto informativo "La consulta ginecológica" (1991), editado por el Ministerio de Sanidad y Consumo, el Ministerio de Asuntos Sociales y el Instituto de la Mujer. 